\title{
Intrahepatic Cholangiocarcinoma with Sarcomatous Changes
}

\author{
Yoshiyuki Inoue Alan T. Lefor Yoshikazu Yasuda \\ Department of Surgery, Jichi Medical University, Shimotsuke, Japan
}

\section{Key Words}

Intrahepatic cholangiocarcinoma - Sarcomatous change

\begin{abstract}
A 61-year-old Japanese male was seen at an outside hospital with abdominal pain and distention. An intraabdominal mass was found on abdominal computed tomography (CT), and he was referred for further evaluation. He was afebrile, with mild tenderness in the upper abdomen and massive distention. Routine blood work showed severe inflammation and anemia. Repeat CT showed a large solitary heterogeneous mass, $25 \mathrm{~cm}$ in diameter, in the left upper quadrant with evidence of intratumoral bleeding and irregular enhancement peripherally. There was no clear distinction between the mass and the peripheral organs. Surgery was performed with persistent anemia and exacerbation of abdominal pain. Lateral segmentectomy, total gastrectomy, cholecystectomy and partial resection of the diaphragm and parietal peritoneum were performed. The final diagnosis was cholangiocarcinoma with sarcomatous changes. On the 16th postoperative day a CT scan revealed recurrent tumor. The patient died on the 34th postoperative day from rapid tumor progression.
\end{abstract}

\section{Introduction}

Epithelial tumors with sarcomatous features are occasionally observed in various organs, including the liver [1]. Most sarcomatous carcinomas in the liver are regarded as sarcomatous differentiation from hepatocellular carcinoma, and their clinical characteristics and pathologic significance have been satisfactorily elucidated [2]. Intrahepatic cholangiocarcinoma with sarcomatous changes is very rare. Sarcomatous transformation has been reported in about $3.9-9.4 \%$ of hepatocellular carcinomas at autopsy and in about $4.5 \%$ of cholangiocarcinomas [3]. Clinical as well as imaging and pathologic information regarding this tumor are still limited. Preoperatively, it may be difficult to establish the definitive diagnosis. Resection is important for prolongation of survival. The optimal treatment of these rare lesions is unclear, partly because of the typically aggressive clinical course. The present case demonstrates some typical features associated with these rare tumors. 


\section{Case Report}

A 61-year-old Japanese male was seen at an outside hospital with abdominal pain and distention. An intraabdominal mass was found on abdominal computed tomography (CT), and he was referred for further evaluation. Both parents had died of hepatic cirrhosis. On physical examination, he was afebrile, with mild tenderness in the upper abdomen and with massive distention. Routine blood tests

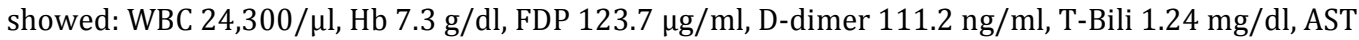
$35 \mathrm{mU} / \mathrm{ml}$, LDH $810 \mathrm{mU} / \mathrm{ml}$, ALP $392 \mathrm{mU} / \mathrm{ml}$, ChE $99 \mathrm{mU} / \mathrm{ml}$, CRP $18.28 \mathrm{mg} / \mathrm{dl}$, HBs antigen negative, $\mathrm{HCV}$ antibody positive, CEA $1.2 \mathrm{ng} / \mathrm{ml}$, and CA19-9 $5 \mathrm{U} / \mathrm{ml}$. Repeat CT showed a large solitary heterogeneous mass, $25 \mathrm{~cm}$ in diameter, in the left upper quadrant with evidence of intratumoral bleeding and irregular enhancement peripherally (arrowhead, fig. 1). There was no clear distinction between the mass and the left lateral segment of the liver. The anterior wall of the stomach was diffusely adherent to the posterior side of the mass. The left side of the diaphragm was also invaded (arrowhead, fig. 2). The tumor seemed to originate from the gastric wall and was suspected to be a gastrointestinal stromal tumor. Needle biopsy revealed only a few spindle-shaped atypical cells.

Surgery was performed due to persistent anemia and exacerbation of abdominal pain. Intraoperative findings revealed that the tumor was densely adherent to the lateral segment of the liver, gallbladder, anterior wall of the stomach, left side of the diaphragm and parietal peritoneum. Lateral segmentectomy, total gastrectomy, cholecystectomy and partial resection of the diaphragm and parietal peritoneum were performed, with a Roux-en-Y reconstruction. The tumor measured $20 \times 15 \times 8 \mathrm{~cm}$. Hematoxylin and eosin staining of histologic sections of the resected specimen showed a malignant neoplasm with undifferentiated spindle cells. Malignant cells with glandular formations similar to intrahepatic bile ducts showed a transition from a differentiated component to an undifferentiated one between the tumor and the liver. Tumor cells invaded the intrahepatic bile duct and replaced normal bile duct epithelium. The carcinomatous component $(<10 \%$ of the cells) stained positive for cytokeratin-7 and cytokeratin-19, but negative for hepatocytes. The sarcomatous component ( $\sim 90 \%$ of the cells) stained positive for vimentin and keratin-1, but negative for cytokeratin-7, cytokeratin-19 and hepatocytes. The adenocarcinoma appeared to be derived from the intrahepatic bile duct with transformation to sarcomatous cells. The final diagnosis was cholangiocarcinoma with sarcomatous changes. On the 16th postoperative day, a CT scan revealed recurrent tumor. The patient was discharged from the hospital for supportive care and died on the 34th postoperative day from rapid tumor progression.

\section{Discussion}

Sarcomatous transformation has been reported to occur in a hepatocellular carcinoma previously treated with transcatheter arterial chemoembolization [3]. There have been no reports concerning the relationship between sarcomatous cholangiocarcinoma and anticancer therapy. Nakajima et al. reported 7 cases of cholangiocarcinoma with sarcomatous changes with a median age of 69 years and abdominal pain as the main presenting symptom [4]. There were no disease-specific findings based on serum chemistry. Abdominal CT showed low-density lesions enhanced at the periphery resembling ordinary cholangiocarcinoma, which were difficult to differentiate by imaging alone. In the present case, a solid lesion and a cystic lesion were found on CT, suggesting a fragile tumor such as a gastrointestinal stromal tumor. Immunohistochemical studies are necessary to establish the diagnosis. Most previously reported cases showed cytokeratin and vimentin expression in the sarcomatous component characteristic of epithelial derivation, as seen in the present case [3-5]. Gupta et al. reported cytomorphological features of cholangiocarcinoma with sarcomatous changes, with spindle-shaped cells, scanty cytoplasm and hyperchromatic nuclei [6]. Although these are useful for preoperative diagnosis, needle biopsy may be contraindicated with the risk of intratumor hemorrhage and peritoneal dissemination, as in our case. 
It has been reported that the prognosis for cholangiocarcinoma with sarcomatous changes is worse than that for typical cholangiocarcinoma [3]. The factors that account for the poor prognosis may be ascribed to the intrahepatic development, especially the high potential of the sarcomatous component to metastasize [3]. Kaibori et al. reported that the survival rate of patients with surgically resected disease was significantly higher than that in patients without resection [5]. There is however no information available about the optimal adjuvant treatment after resection. Malhotra et al. reported combination chemotherapy with gemcitabine and cisplatin as a potential treatment for controlling disease progression [3]. In our case, there was no time to give adjuvant therapy because of rapid recurrence and general deterioration.

Cholangiocarcinoma with sarcomatous changes is an extremely rare tumor composed of mixed malignant epithelial and mesenchymal cells. The outcome of this case shows that this neoplasm generally pursues a highly aggressive and malignant clinical course, with rapid growth and wide local infiltration, leading to poor prognosis. Radical surgery with adjuvant chemotherapy and close follow-up may be important in the effective management of this disease.

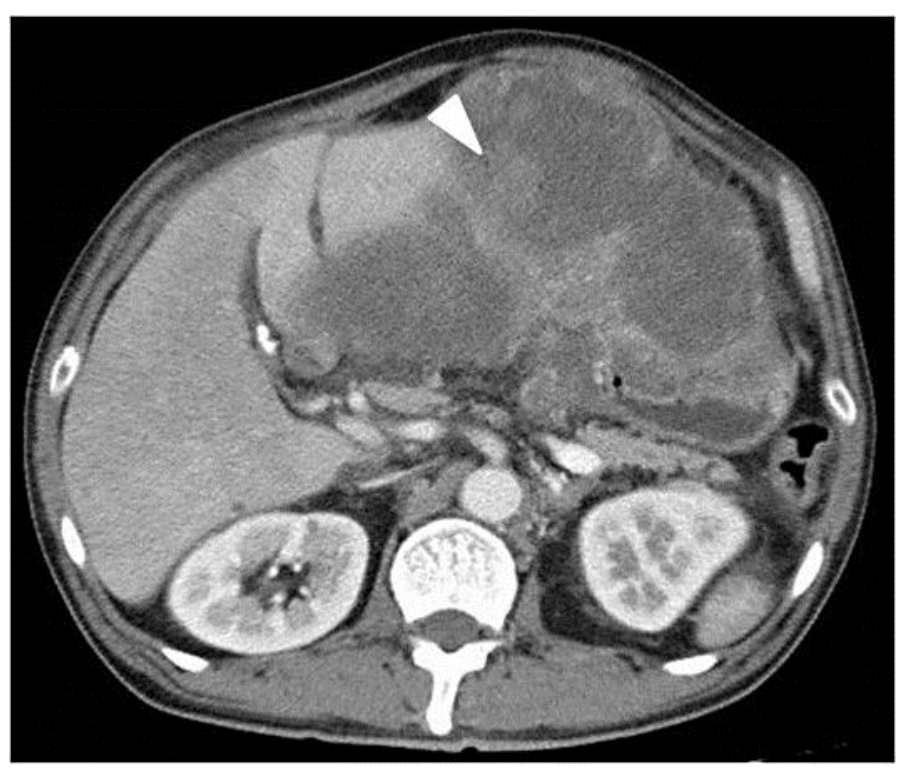

Fig. 1. CT scan of the abdomen showed a tumor with solid and cystic components. The arrowhead shows intratumoral bleeding. 


\begin{tabular}{|c|c|c|c|}
\hline $\begin{array}{l}\text { Case Reports in } \\
\text { Gastroenterology }\end{array}$ & $\begin{array}{l}\text { Case Rep Gastroenterol 2012;6:1-4 } \\
\text { DOI: } 10.1159 / 000335883\end{array}$ & $\begin{array}{l}\text { Published online: } \\
\text { January 4, } 2012\end{array}$ & $\begin{array}{l}\text { @ } 2012 \text { S. Karger AG, Basel } \\
\text { ISSN } 1662-0631 \\
\text { www.karger.com/crg }\end{array}$ \\
\hline
\end{tabular}

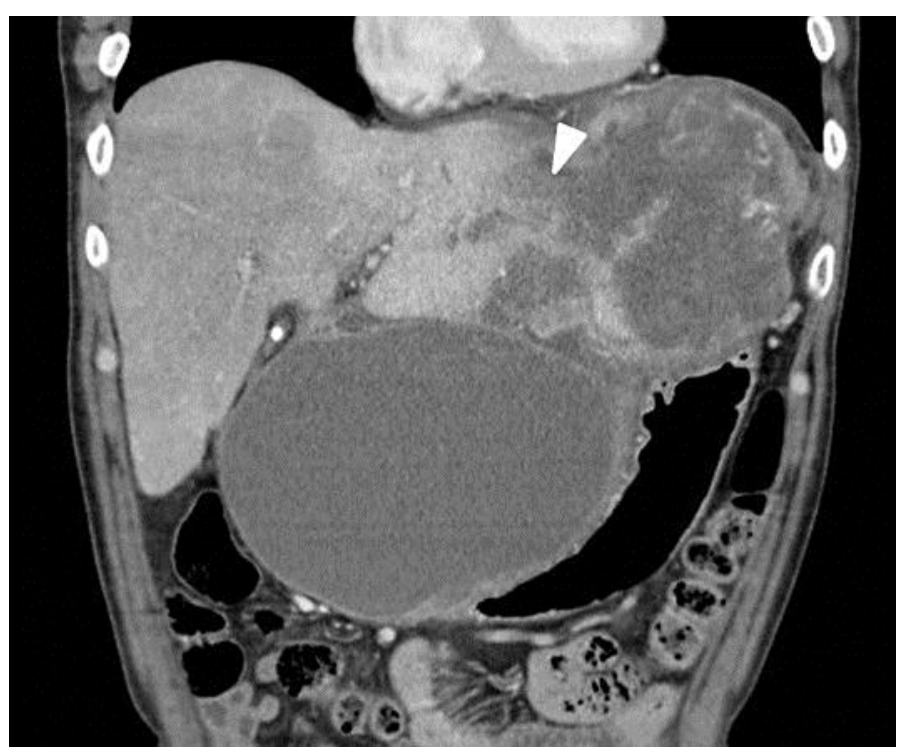

Fig. 2. CT demonstrated no sharp delineation between the tumor and the lateral lobe of the liver (arrowhead). The tumor invaded the diaphragm.

\section{References}

1 Maeda T, Adachi E, Kajiyama K, Takenaka K, Sugimachi K, Tsuneyoshi M: Spindle cell hepatocellular carcinoma. A clinicopathologic and immunohistochemical analysis of 15 cases. Cancer 1996;77:51-57.

-2 Kakizoe S, Kojiro M, Nakashima T: Hepatocellular carcinoma with sarcomatous change. Clinicopathologic and immunohistochemical studies of 14 autopsy cases. Cancer 1987;59:310-316.

-3 Malhotra S, Wood J, Mansy T, Singh R, Zaitoun A, Madhusudan S: Intrahepatic sarcomatoid cholangiocarcinoma. J Oncol 2010;2010:701476.

-4 Nakajima T, Tajima Y, Sugano I, Nagao K, Kondo Y, Wada K: Intrahepatic cholangiocarcinoma with sarcomatous change. Clinicopathologic and immunohistochemical evaluation of seven cases. Cancer 1993;72:1872-1877.

5 Kaibori M, Kawaguchi Y, Yokoigawa N, et al: Intrahepatic sarcomatoid cholangiocarcinoma. J Gastroenterol 2003;38:1097-1101.

6 Gupta K, Powari M, Dey P: Sarcomatous cholangiocarcinoma. Diagn Cytopathol 2003;28:168-169. 